\title{
Excited state vibrational coherence in a binuclear metal adduct: wave packet phase dependant molecular fragmentation under variation of ligand size
}

\author{
Sebastian Volker Kruppa ${ }^{1, *}$, Florian Bäppler ${ }^{2}$, Christof Holzer $^{3}$, Fabian Dietrich ${ }^{1}$, \\ Rolf Diller ${ }^{2}$, Wim Klopper $^{3}$, and Christoph Riehn ${ }^{1,4, *}$ \\ ${ }^{1}$ TU Kaiserslautern, Chemistry Dept., Erwin-Schrödinger Str. 52-54, 67663 Kaiserslautern, Germany \\ ${ }^{2}$ TU Kaiserslautern, Physics Dept., Erwin-Schrödinger Str. 46, 67663 Kaiserslautern, Germany \\ ${ }^{3}$ Karlsruhe Institute of Technology, Institute of Physical Chemistry, Fritz-Haber Weg 2, 76131 \\ Karlsruhe, Germany \\ ${ }^{4}$ Landesforschungszentrum OPTIMAS, Erwin-Schrödinger Str. 46, 67663 Kaiserslautern, Germany
}

\begin{abstract}
Ultrafast vibrational coherence with metal-metal stretching mode character is observed for phosphine-bridged $\mathrm{d}^{10}-\mathrm{d}^{10}$ complexes of different size, containing the binuclear $\left[\mathrm{Ag}_{2} \mathrm{Cl}\right]^{+}$chromophore in an ion trap. Fragmentation pathways are governed by the wave packet's phase.
\end{abstract}

\section{Introduction}

Ultrafast photo-induced dynamics of ligand-stabilized transition metal complexes is a rapidly advancing field due to their widespread applications e.g. in organic light emitting diodes (OLEDs), sensors, light harvesting (dye-sensitized solar cells), photocatalysis and medicine. Thus, many femto/picosecond-resolved methods are currently employed such as transient optical spectroscopy, 4D electron diffraction as well as front-edge X-ray techniques. However, gas phase studies giving insight into the intrinsic dynamics in the absence of a surrounding medium are still scarce.

Our gas phase approach is based on time-resolved photodissociation ( $\tau$-PD) action spectroscopy [1-3] of mass-selected, ion-trapped molecular systems, probing their electronic dynamics by way of transient fragmentation patterns as a function of pump-probe delay. Besides information on excited state kinetics, we focus on the question of how a subtle quantum phenomenon such as vibrational coherence is influenced by structural and electronic parameters, modulating cooperative metal-metal interaction.

Therefore, we studied $\mathrm{d}^{10}-\mathrm{d}^{10}$ complexes by example of the phosphine-ligand bridged $\left[\mathrm{Ag}_{2}(\mathrm{Cl})\right]^{+}$scaffold (Fig. 1), providing opportunities for chemical modifications e.g. by variation of the ligand size. We compare the wave packet dynamics of complexes with the bulky $\mathrm{L}^{\mathrm{Cy}}$ ligands $\left(\mathbf{2}^{+}, 149\right.$ atoms, $\mathrm{R}=\mathrm{C}_{6} \mathrm{H}_{11}$, cyclohexyl) to those of the smaller $\mathrm{L}^{\mathrm{Me}}$ ligands $\left(\mathbf{1}^{+}, 45\right.$ atoms, $\mathrm{R}=\mathrm{CH}_{3}$, methyl) and observe a correlation between the wave

\footnotetext{
*Corresponding authors: kruppa@chemie.uni-kl.de, riehn@chemie.uni-kl.de
} 
packet's phase and fragment type and an (at first glance) astonishing slow-down of vibrational dynamics as discussed in the next section.

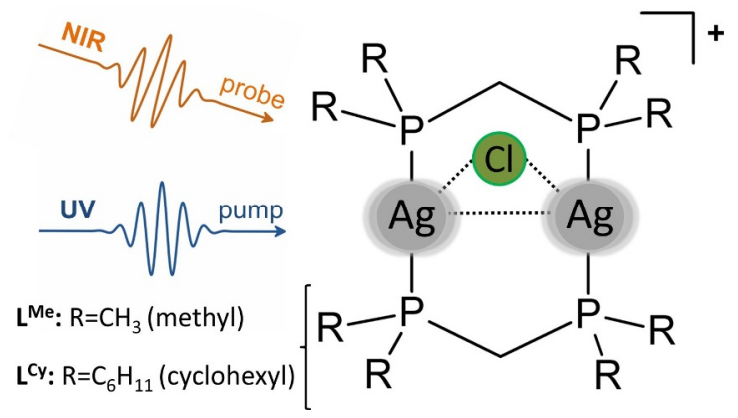

Fig. 1. Schematic structures of complexes $\left[\mathrm{Ag}_{2}(\mathrm{Cl})\left(\mathrm{L}^{\mathrm{Me}}\right)_{2}\right]^{+}, \mathbf{1}^{+}$and $\left[\mathrm{Ag}_{2}(\mathrm{Cl})\left(\mathrm{L}^{\mathrm{Cy}}\right)_{2}\right]^{+}, \mathbf{2}^{+}$, respectively $\left(\mathrm{L}^{\mathrm{Me}}=\right.$ bis(dimethylphosphino)methane, $\mathrm{L}^{\mathrm{Cy}}=$ bis(dicyclohexy/phosphino)methane). For $\tau$-PD experiment UV-pump $(280 \mathrm{~nm})$ and NIR-probe $(1150 \mathrm{~nm})$ pulses were used.

\section{Results and Discussion}

The experimental setup and methodology is described elsewhere [1-3]. The chloride adducts $\left[\mathrm{Ag}_{2}(\mathrm{Cl})\left(\mathrm{L}^{\mathrm{Me}}\right)_{2}\right]^{+}\left(\mathbf{1}^{+}\right)$and $\left[\mathrm{Ag}_{2}(\mathrm{Cl})\left(\mathrm{L}^{\mathrm{Cy}}\right)_{2}\right]^{+}\left(\mathbf{2}^{+}\right)$where generated by electrospray ionization from acetonitrile sample solutions, isolated and identified by mass analysis via their characteristic isotope patterns $(\mathrm{m} / \mathrm{z}=521$ and 1065 , resp. nominal masses). The static photo-induced dissociation (PD) spectra show a band at $\lambda_{\max } \sim 280 \mathrm{~nm}$ and a plateau-like form centered at $\sim 260 \mathrm{~nm}$ for $\mathbf{1}^{+}$and $\mathbf{2}^{+}$, resp. Based on calculations (PBE0-D3(BJ)/ev $G W$ $\mathrm{BSE} / \mathrm{def} 2-\mathrm{SVPD})$ [4], using $\mathbf{1}^{+}$as model system, we assign these excitations to HOMO-1 $\rightarrow$ LUMO ( $\left.{ }^{1} \mathrm{XMCT}\right)$ transitions with chloride-to-metal-metal charge transfer character [1]. Hereby a contraction of the Ag-Ag distance by $\sim 0.1 \AA$ is predicted (CC2/def2-TZVPP), indicating a strengthening of Ag-Ag interaction in the electronically excited state.

The $\tau$-PD signals of $\mathbf{1}^{+}$at positive time delay show an increase in the channels corresponding to neutral loss of $\mathrm{Cl}, \mathrm{AgCl}$ and $\mathrm{AgCl}+\mathrm{L}^{\mathrm{Me}}$, resp., and a concomitant decrease in intensity for $-\mathrm{L}^{\mathrm{Me}}$ fragment species (Fig. 2 c). The early decay dynamics of $\mathbf{1}^{+}$ with time constants $0.2-0.5 / 4-6$ ps (Fig. 2) is very similar compared to that of $2^{+}$with $0.2-$ 0.4 / 3-4 ps [1]. Interestingly, a correlation between specific fragment ions ( $\mathrm{Cl}$ loss vs loss of $\mathrm{L}^{\mathrm{Me}}+\mathrm{AgCl}$ ) and the phase of the wave packet is observed, as evident by strongly damped (damping constant $\sim 0.1-0.5 \mathrm{ps}$ ) oscillatory components in their $\tau$-PD signals (Fig. $2 \mathrm{a}, \mathrm{b}$ ), exhibiting a relative phase difference of $\sim \pi$. A similar result was obtained for the $\left[\mathrm{Ag}_{2}(\mathrm{Cl})\left(\mathrm{L}^{\mathrm{Cy}}\right)_{2}\right]^{+}$molecular target displaying an analogous relation mainly for the fragmentations $-\mathrm{HCl} /-\mathrm{Cl}^{-}$vs loss of $\mathrm{L}^{\mathrm{Cy}}$. Note that the parallel investigation of $\left[\mathrm{Ag}_{2}(\mathrm{Cl})\left(\mathrm{L}^{\mathrm{Cy}}\right)_{2}\right]^{+}$in condensed phase (acetonitrile) by transient absorption does not yield detectable vibrational wave packet activity.

Since the largest structural changes upon photoexcitation correspond to the decrease of metal distance $(\mathrm{d}(\mathrm{Ag}-\mathrm{Ag})=-3.6 \%)$ and the bending of $\mathrm{P}-\mathrm{Ag}-\mathrm{P}$ angle $(\Varangle(\mathrm{P}-\mathrm{Ag}-\mathrm{P})=+3.3 \%))$, this observation strengthens our previous assignment for the vibrational coherence to contain mainly Ag-Ag stretching mode character [1].

Intriguingly, the oscillation frequency for $\mathbf{1}^{+}\left(43-60 \mathrm{~cm}^{-1}\right)$ is significantly smaller than that for $\mathbf{2}^{+}\left(72-78 \mathrm{~cm}^{-1}\right)$. Since a higher frequency value would be expected for $\mathbf{1}^{+}$ $\left(\operatorname{mass}\left(\mathrm{L}^{\mathrm{Me}}\right)<<\operatorname{mass}\left(\mathrm{L}^{\mathrm{Cy}}\right)\right)$ this indicates a significantly weaker $\mathrm{Ag}-\mathrm{Ag}$ bonding interaction for $\mathbf{1}^{+}$compared to $\mathbf{2}^{+}$in the excited state. Therefore, we assume that in the excited state metallophilic Ag-Ag interaction is subtly influenced by the ligand moieties (methyl vs cyclohexyl), presumably due to polarization or electron inductive effects. 

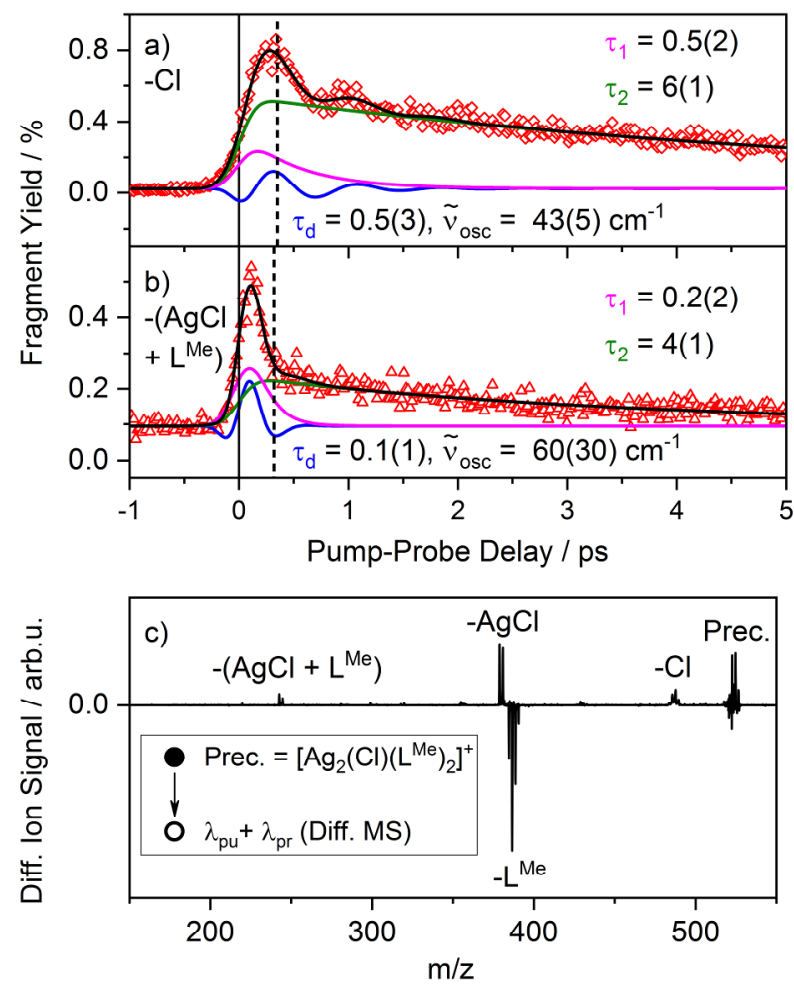

Fig. 2. Fragment-specific transients of $\left[\mathrm{Ag}_{2}(\mathrm{Cl})\left(\mathrm{L}^{\mathrm{Me}}\right)_{2}\right]^{+}\left(\lambda_{\text {pump: }}: 280 \mathrm{~nm} / \lambda_{\text {probe: }} 1150 \mathrm{~nm}\right)$ corresponding to a) loss of $\mathrm{Cl}$ atom and b) loss of $\mathrm{AgCl}+\mathrm{L}^{\mathrm{Me}}$, respectively. Kinetic fits (black lines) and individual decay contributions associated with time constants $\tau_{1}$ (magenta), $\tau_{2}$ (green), oscillatory components with coherence decay times $\tau_{\mathrm{d}}$ (blue) and oscillation frequencies $v_{\text {osc }}$ are displayed. The fit function corresponds to a convolution of a Gaussian (FWHM $\sim 300 \mathrm{fs}$ ) with a sum of a biexponential decay function and a damped sine function. c) Difference mass spectrum (Diff. MS) obtained by subtracting the mass spectrum at negative pump-probe delay $(\sim-(1-0.3)$ ps) from the spectrum at positive delay $(\sim 0.2-0.6 \mathrm{ps})$. Positive and negative Diff. MS signals correspond to an enhancement and depletion, respectively. Fragment mass peaks are labelled according to their neutral losses relative to the intact precursor ion (Prec.).

Further, we conclude that the cross section for probe absorption, and thus specific excitation, is modulated by the vibrational wave packet at the classical turning points of the Ag-Ag oscillation, leading to the phase-dependent branching ratios of molecular fragmentation channels. These results open new perspectives in leveraging vibrational coherence for chemical processes and should therefore stimulate further experimental and theoretical studies.

\section{References}

1. S. V. Kruppa, F. Bäppler, C. Holzer, W. Klopper, R. Diller, C. Riehn, J. Phys. Chem. Lett., 9, 804-810 (2018)

2. S. V. Kruppa, F. Bäppler, W. Klopper, S. P. Walg, W. R. Thiel, R. Diller, C. Riehn, Phys. Chem. Chem. Phys., 34, 22785-22800 (2017)

3. D. Imanbaew, J. Lang, M. F. Gelin, S. Kaufhold, M. G. Pfeffer, S. Rau, C. Riehn, Angew. Chem. Int. Ed., 56, 5471-5474 (2017)

4. K. Krause, W. Klopper, J. Comput. Chem., 38, 383-388 (2017) 